\title{
Asociación entre enfermedad de Crohn y colangitis esclerosante primaria en una niña de $\mathbf{1 0}$ años Association between Crohn's disease and primary sclerosing cholangitis in a 10 year old girl
}

\author{
Dra. Ana Muñoz Lozón ${ }^{a}$, Dra. Cristina Iglesias Blázquez ${ }^{a}$ Dra. Cristina Menéndez Arias ${ }^{a}$ y \\ Dra. Patricia Dominguez Sánchez ${ }^{a}$
}

\begin{abstract}
RESUMEN
Niña de 10 años con diarrea, abdominalgia, pérdida de peso y febrícula de un mes y medio de evolución. Los hallazgos analíticos y ecográficos hicieron sospechar una enfermedad inflamatoria intestinal. La endoscopía y la histología mostraron hallazgos compatibles con la enfermedad de Crohn. Se inició un tratamiento con mesalazina y nutrición enteral exclusiva, y se añadieron corticoides, inmunosupresores y ácido ursodesoxicólico por la persistencia de colestasis e hipergammaglobulinemia. La colangiorresonancia y la biopsia hepática confirmaron el diagnóstico de colangitis esclerosante primaria concomitante.

La asociación entre la enfermedad de Crohn y la colangitis esclerosante primaria es muy poco frecuente; predomina en varones de entre los 20 y los 40 años de edad; y presenta una gran variabilidad clínica. El diagnóstico de confirmación requiere la realización de una colangiorresonancia o colangiopancreatografía retrógrada endoscópica. El pronóstico es malo y no existe ningún tratamiento capaz de frenar la progresión de la enfermedad.

Palabras clave: enfermedad de Crohn, colangitis esclerosante primaria, enfermedades inflamatorias intestinales.
\end{abstract}

\section{ABSTRACT}

A 10 year old girl with diarrhea, abdominal pain, weight loss and fever of one month and a half of evolution. Analytical and sonographic findings raised the possibility of inflammatory bowel disease. Endoscopy and histology showed findings consistent with Crohn's disease. Treatment was initiated with mesalazine and exclusive enteral nutrition. Later corticosteroid treatment, immunosuppressive drugs and ursodeoxycholic acid were added due to cholestasis and persistent hypergammaglobulinemia. Magnetic resonance cholangiography and liver biopsy confirmed the diagnosis of concomitant primary sclerosing cholangitis.

The association between Crohn's disease and primary sclerosing cholangitis is rare, predominantly in males

a. Servicio de Pediatría del Complejo Asistencial de León. España.

Correspondencia:

Dra. Ana Muñoz Lozón, amunozlozon@gmail.com

Financiamiento: Ninguno.

Conflicto de intereses: Ninguno que declarar.

Recibido: 21-10-2015

Aceptado: 21-12-2015 between 20 and 40 years old and it presents a great clinical variability. The confirmation of the diagnosis requires magnetic resonance cholangiography or endoscopic retrograde cholangiopancreatography. The prognosis is poor and there is no treatment to slow the progression of the disease.

Key words: Crohn's disease, primary sclerosing cholangitis, inflammatory bowel diseases.

http:/ /dx.doi.org/10.5546/aap.2016.e187

\section{INTRODUCCIÓN}

La enfermedad de Crohn (EC) es una enfermedad inflamatoria intestinal (EII) que afecta a cualquier parte del tracto gastrointestinal, desde la boca hasta el ano, con mayor afectación del íleon y del colon. Se caracteriza por una inflamación granulomatosa, transmural y discontinua. ${ }^{1}$ Su etiología es desconocida, aunque se considera que influyen factores genéticos y ambientales en su etiopatogenia.

\section{CASO CLÍNICO}

Niña de 10 años con diarrea sanguinolenta de un mes y medio de evolución, pérdida de peso de $4 \mathrm{~kg}$, anorexia y febrícula vespertina ocasional. Se asoció abdominalgia periumbilical en las últimas 24 horas. Antecedentes personales y familiares sin interés.

Exploración física: peso de 28,5 kg (Pc 10-25), talla de 138 cm (Pc 10-25). Escaso panículo adiposo. Dolor abdominal con la palpación profunda en la región periumbilical. No se palparon masas ni visceromegalias. Sin otros hallazgos en la exploración física.

Se decidió el ingreso para su estudio. Presentó un hemograma normal, sin signos de anemia ni trombocitosis, velocidad de sedimentación globular (VSG) de $96 \mathrm{~mm}$, bioquímica sanguínea con elevación de parámetros inflamatorios (proteína C reactiva $-\mathrm{PCR}-18,8 \mathrm{mg} / \mathrm{L}$; orosomucoide: $154 \mathrm{mg} / \mathrm{dl}$ ), hipertransaminasemia (aspartato-aminotransferasa -AST-: $57 \mathrm{UI} / \mathrm{L}$; alanino-aminotransferasa -ALT-: $221 \mathrm{UI} / \mathrm{L}$; 
fosfatasa alcalina -FA-: 992 UI/L; gammaglutamil transpeptidasa -GGT-: 309 UI/L); IgG: $1811 \mathrm{mg} / \mathrm{dl}$; albúmina: 4,09 $\mathrm{g} / \mathrm{dl}$; prealbúmina y proteína transretinol descendidas $(9,9 \mathrm{mg} / \mathrm{dl}$ y $1,67 \mathrm{mg} / \mathrm{dl}$, respectivamente). Además, se observó una calprotectina fecal de $643 \mathrm{mg} / \mathrm{kg}$ y el estudio infeccioso de heces fue negativo.

El estudio de inmunidad presentó positividad para anticuerpos anticitoplasma de neutrófilo 1/2560 patrón c-ANCA con especificidad para anticuerpos anticatepsina G y antilisozima. El estudio de histocompatibilidad muestra HLADQ (DQ2, DQ-) y HLA-DR (DR17, DR52, DR7 y DR53).

En la ecografía abdominal, se observó un engrosamiento y un aumento de la vascularización de la pared del colon, desde la válvula ileocecal hasta el colon descendente, que respetaba el sigma (Figura 1). Se identificó, además, una formación quística de $6 \times 3,3 \mathrm{~cm}$, que parecía hepática, aunque no se podía descartar que fuera subcapsular.

Se realizó una endoscopía digestiva, en la que se observó un aspecto eritematoso del colonsigma, sin ulceraciones, y se confirmaron, en el estudio anatomopatológico, signos de ileítis crónica activa y alteraciones histológicas en el colon. La enterorresonancia demostró una amplia afectación del colon, íleon terminal y yeyuno

FIgURA 1. Ecografía abdominal. Engrosamiento y aumento de la vascularización de la pared del colon

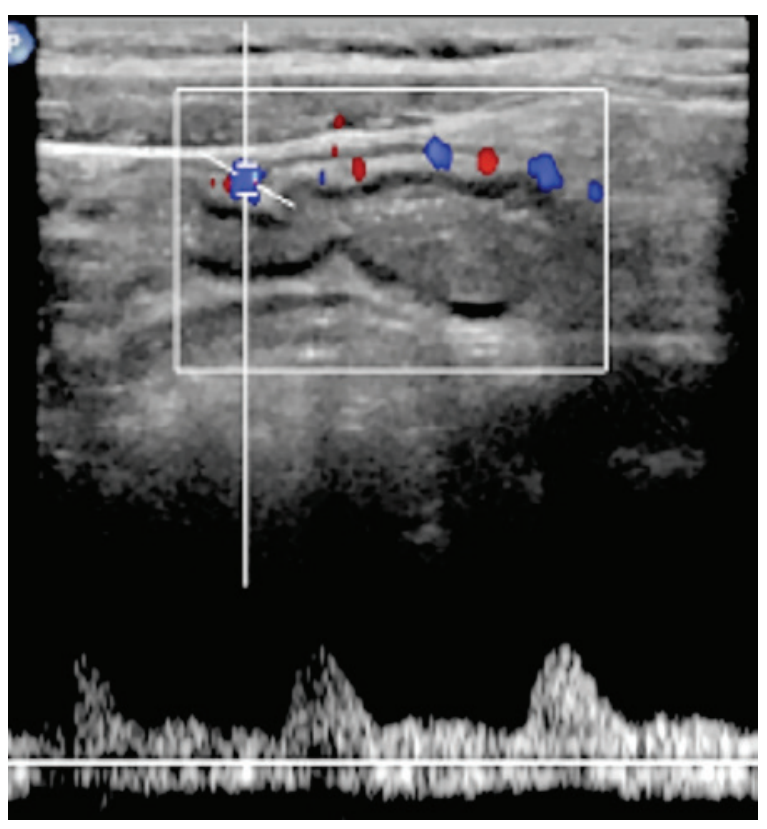

en fase activa. Todos estos datos sugerían la presencia de una EII tipo EC.

Hasta la confirmación histológica (Figura 2), se inició un tratamiento con mesalazina oral ( $3 \mathrm{~g})$ y rectal $(1 \mathrm{~g})$, y nutrición enteral exclusiva con fórmula especial de EII, con lo que se consiguió una discreta mejoría clínica, pero persistían los parámetros inflamatorios analíticos. Tres semanas más tarde, se comenzó un tratamiento con corticoterapia oral $(2 \mathrm{mg} / \mathrm{kg})$ y azatioprina (3 mg/ $\mathrm{kg}$ ), con disminución de la sintomatología y reactantes de fase aguda.

Debido a la hipertransaminasemia, la colestasis y la hipergammaglobulinemia mantenida, se sospechó la posibilidad de una lesión hepática concomitante, por lo que se decidió la realización de una colangiorresonancia y una biopsia hepática. La resonancia magnética mostró una dudosa estenosis en la confluencia de los conductos hepáticos principales y hepático común, y una lesión quística lobulada extrahepática (Figura 3). En la biopsia, se observaron alteraciones histológicas compatibles con colangitis esclerosante primaria (CEP). Se inició un tratamiento con $10 \mathrm{mg} / \mathrm{kg}$ de ácido ursodesoxicólico (que coincidió con el inicio de la terapia combinada de azatioprina y corticoide), con normalización de las cifras de FA, GGT, AST, ALT e IgG.

La paciente tuvo un brote de EII a los 12 meses del diagnóstico, que fue corticorresistente, por lo que se inició un tratamiento biológico con infliximab, que requirió intensificar el tratamiento hasta $10 \mathrm{mg} / \mathrm{kg} / \mathrm{dosis}$. Posteriormente, tuvo buen control sin recaídas durante 2 años. Presentó otro brote leve con

FIgURA 2. Imagen histológica de íleon distal. Mucosa de intestino delgado con afectación parcheada de la lámina propia por un infiltrado inflamatorio crónico linfoplasmocitario con imágenes de permeación del epitelio por neutrófilos (signos de criptitas) y presencia de algún absceso críptico aislado

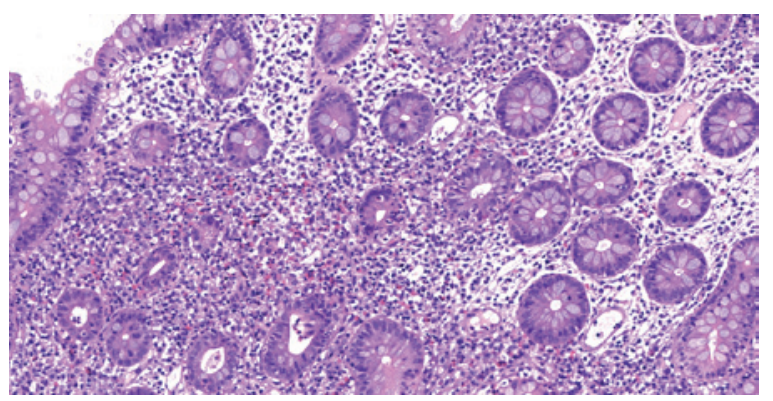


elevación de calprotectina fecal y mínima afectación analítica, que coincidió con niveles bajos de anticuerpos monoclonales. En la colangiorresonancia realizada durante este brote, se observó un empeoramiento radiológico (Figura 4); los estudios de función hepática no mostraron colestasis.

\section{COMENTARIOS}

La CEP es una enfermedad colestásica crónica, caracterizada por inflamación y fibrosis progresiva, intra- y extrahepática, que conlleva estenosis multifocal y, frecuentemente, evoluciona a cirrosis biliar y falla hepática. Se trata de un patología poco frecuente, con una incidencia en la población total en el norte de Europa de 0,9-1,3/100 000 habitantes/año; en niños, es $<20 \%$ de la encontrada en adultos. ${ }^{2}$

Su etiología es desconocida. Su asociación con otras enfermedades autoinmunes sugiere una base genética e inmunológica. Se ha detectado una alta implicación de los haplotipos HLA-DR3, DRw52a y DR4, y de anticuerpos antinucleares (24\%-53\%), antimúsculo liso (13\%-20\%) y anticitoplasmáticos perinucleares p-ANCA (65\%-88\%). ${ }^{3}$ En pacientes con EII y CEP, se encuentra una alta prevalencia de anticuerpos

Figura 3. Colangiorresonancia magnética nuclear. Estenosis en la confluencia de los conductos hepáticos principales y origen del conducto hepático común (imagen visualizada únicamente en el plano coronal pero no en el plano axial)

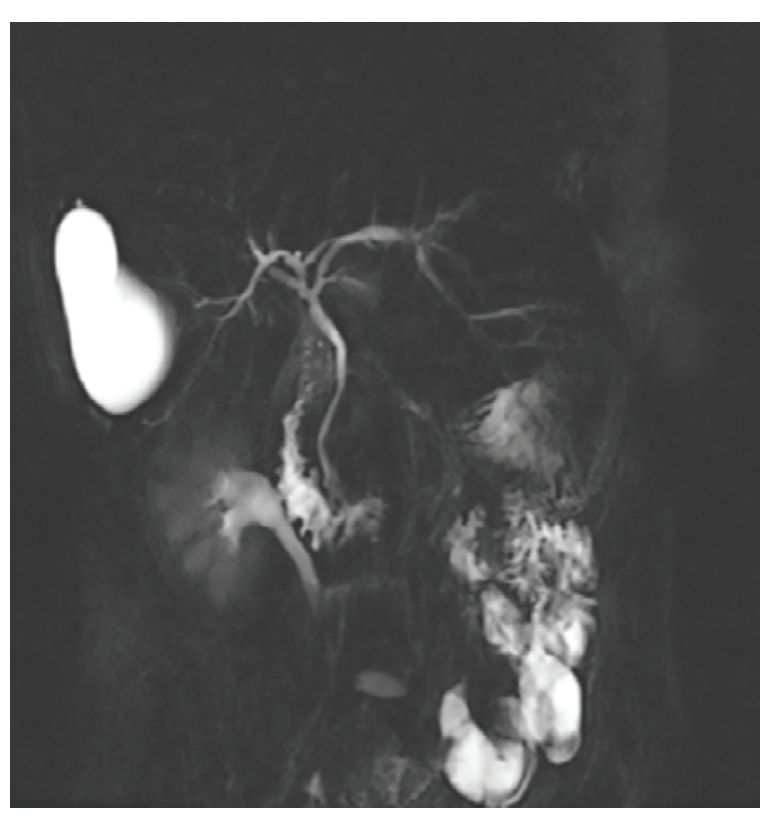

p-ANCA, y es más frecuente en los pacientes con colitis ulcerosa (CU) $(23 \%-80 \%)$ que en los pacientes con EC $(0 \%-40 \%) .{ }^{4}$ Aunque el patrón de inmunofluorescencia que se observa en la EII es perinuclear en la mayoría de los casos, puede encontrarse un patrón citoplasmático hasta en un $38 \%$, sobretodo asociado a la EC. ${ }^{5}$ El significado de este hallazgo es controvertido, aunque algunos autores señalan que la presencia de ANCA o su título podrían relacionarse con la actividad de la enfermedad o, incluso, se postula la posible relación de estos anticuerpos con la afectación de colon descendente y recto. ${ }^{4}$

La CEP es la manifestación hepática más frecuentemente encontrada en los pacientes con EII, con una prevalencia estimada del 2\%-7,5\% de los pacientes con $\mathrm{CU}$; su asociación es infrecuente en los pacientes con EC $(1,4 \%-3,4 \%) .{ }^{3}$ Así, de

FIGURA 4. Última colangiorresonancia magnética nuclear realizada en nuestro paciente. Vía extrahepática: varias estenosis en el trayecto intrapancreático del colédoco y de su origen con dilatación entre ambos segmentos. Estenosis de los conductos hepáticos principales y la unión de ambos, con dilatación del conducto hepático común. Vía intrahepática: evidentes estenosis

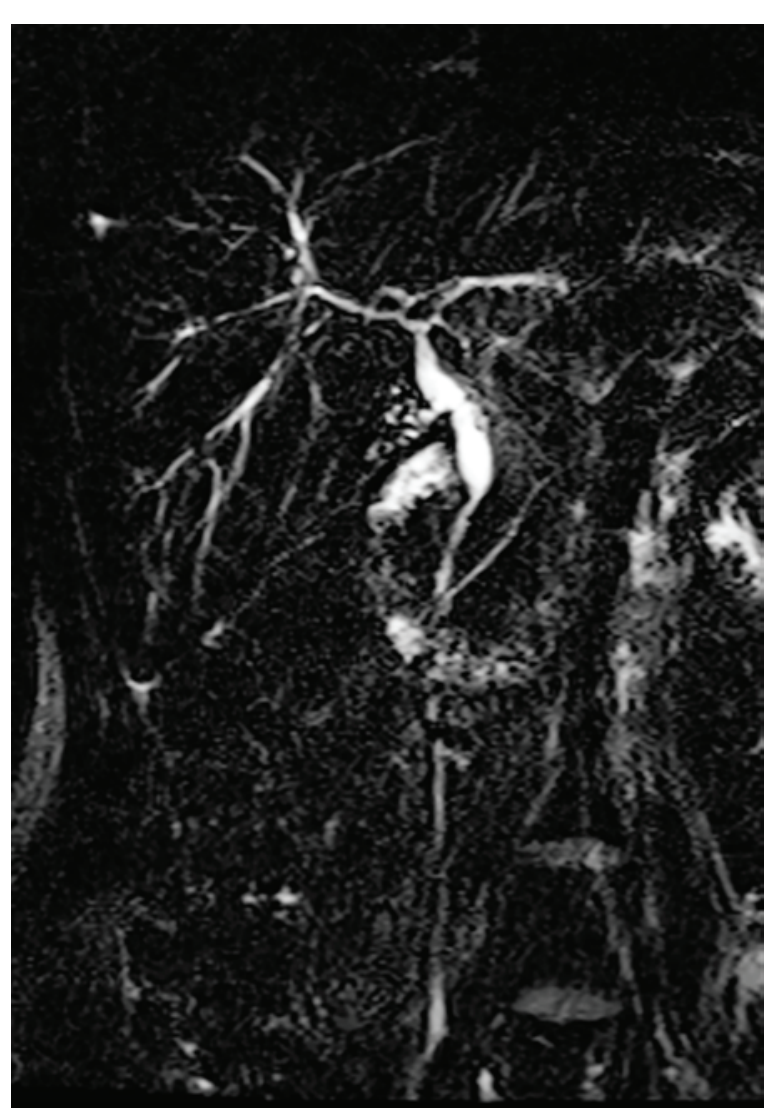


los pacientes con CEP y EII, solo un 10\%-15\% tendrán EC, mientras que el $80 \%$ padecerán $\mathrm{CU}{ }^{2}$ Sin embargo, parece ser que la incidencia de CEP en los pacientes con EC es mayor en los casos de colitis extensiva y se aproxima a la incidencia estimada en la CU. Además, la asociación de EC y CEP es predominante en varones con edades comprendidas entre los 20 y los 40 años. ${ }^{6}$

Aunque la CEP se relaciona con la inflamación intestinal y la extensión de la enfermedad, sigue un curso independiente de ella y puede preceder o aparecer en pacientes con enfermedad inflamatoria intestinal ya diagnosticada. No se ha encontrado correlación entre el grado de afectación hepática y la actividad de la EII.,6 Nuestra paciente, sin embargo, presentó mejoría analítica de la enfermedad hepática y de la enfermedad inflamatoria intestinal y disminución de la sintomatología asociada tras el inicio del tratamiento combinado con corticoterapia sistémica, tratamiento inmunosupresor y ácido ursodesoxicólico. En su evolución, además, se observó un empeoramiento radiológico del arrosariamiento de las vías biliares, que coincidió con un brote leve de su enfermedad de Crohn.

Los síntomas de la CEP asociados a la EC son inespecíficos y variables. Podemos encontrar casos asintomáticos $(15 \%-40 \%)^{6}$ o con ictericia, prurito, dolor abdominal, fiebre, pérdida de peso e, incluso, síntomas de hipertensión portal. Debido a la gran variabilidad clínica presentada, el diagnóstico de CEP en los pacientes con EC constituye un desafío y los síntomas pueden confundirse con otras manifestaciones hepatobiliares de la enfermedad, como la hepatitis autoinmune.

De este modo, debemos sospechar la coexistencia de una CEP en aquellos pacientes con EC y hallazgos de laboratorio compatibles con colestasis. La mayoría de los pacientes presentan elevación de la fosfatasa alcalina (enzima hepática más comúnmente elevada), ${ }^{6}$ con valores de GGT y bilirrubina normales. ${ }^{2}$ La elevación de la fosfatasa alcalina se aprecia en la patología hepatobiliar, aunque también puede darse de forma fisiológica en períodos de crecimiento y de reparación ósea. El aumento de GGT puede ayudar a discernir el origen de la elevación de la fosfatasa alcalina, que es un marcador muy sensible de colestasis prolongada. En nuestro caso, además de la FA, se elevaron la GGT, AST y ALT. El diagnóstico definitivo requiere la comprobación de la vía biliar. Se recomienda como técnica inicial la colangiorresonancia magnética con una alta sensibilidad (85\%) y especificidad (100\%) y menos efectos secundarios. La colangiopancreatografía retrógrada endoscópica (CPRE) se utiliza cuando se contempla una actitud terapéutica. ${ }^{2}$ Otras pruebas de imagen, como la tomografía axial computada (TAC), no se recomiendan. La biopsia hepática se reserva para los casos con elevación de enzimas hepáticas y colangiorresonancia normal, para descartar la presencia de una colangitis esclerosante de pequeños canales intrahepáticos. Así mimo, está indicada con el fin de excluir otras enfermedades hepáticas, como la hepatitis autoinmune o síndromes de solapamiento. ${ }^{2}$

El manejo terapéutico de estos pacientes sigue siendo incierto, ya que no hay ningún fármaco que haya demostrado frenar la evolución de la enfermedad ni prolongar la supervivencia. El ácido ursodesoxicólico en dosis bajas consigue mejorar la función hepática y las alteraciones anatomopatológicas, sin influir en la progresión de la enfermedad. ${ }^{3,6}$ En niños con síndrome de solapamiento, IgG elevadas y marcadores autoinmunes, se recomienda una terapia combinada con inmunosupresores y corticoides, que consiguen disminuir la inflamación, aunque no la normalización de las lesiones. El trasplante hepático constituye el único tratamiento eficaz en fase avanzada.

La supervivencia media libre de trasplante en los pacientes con CEP es de 18 años en las formas asintomáticas y de 8,5 años en las formas sintomáticas. ${ }^{7}$ La CEP asociada a la EC tiene mal pronóstico, con una supervivencia media desde el diagnóstico a los 10 años del 65\%. Tras el trasplante hepático, la supervivencia a los 5-10 años es del 70\%-85\%. ${ }^{2,6}$ Las principales causas de muerte son el cáncer colorrectal, colangiocarcinoma o falla hepática. ${ }^{2,6}$ Los pacientes que presentan síntomas en el momento del diagnóstico tienen una peor evolución. ${ }^{6}$ Estudios recientes han encontrado una mayor incidencia de displasia colónica y cáncer colorrectal, además de una aparición más precoz en pacientes con CEP y EEII concomitante..$^{7-9}$

\section{REFERENCIAS}

1. Medina E. Enfermedad inflamatoria intestinal (I): clasificación, etiología y clínica. An Pediatr Contin 2013; 11(2):59-67.

2. Krones E, Graziadei I, Trauner M, Fickert P. Evolving concepts in primary sclerosing cholangitis. Liver Int 2012;32(3):352-69.

3. Montón Rodríguez C, Ferrer Bradley I. Manifestaciones hepáticas en la enfermedad inflamatoria intestinal. Enfermedad Inflamatoria Intestinal al Día 2012;11(1):60-72.

4. Martínez Odriozola P, Lizarralde Palacios E, Gutiérrez 
Macías A. Hepatitis autoinmune asociada a enfermedad de Crohn y anticuerpos anticitoplasma de los neutrófilos con patrón citoplásmico. Gastroenterol Hepatol 2005; 28(5):306-10.

5. IBD Working Group of the European Society for Paediatric Gastroenterology, Hepatology and Nutrition. Inflammatory bowel disease in children and adolescents: recommendations for diagnosis-the Porto criteria. J Pediatr Gastroenterol Nutr 2005;41(1):1-7.

6. Miao XP, Sun XN, Wei H, Ouyang Q. Crohn's disease and primary sclerosing cholangitis: a case report and review of the literature. Intern Med 2012;51(16):2077-81.
7. Parés A. Colangitis esclerosante primaria: diagnóstico, pronóstico y tratamiento. Gastroenterol Hepatol 2011; 34(1):41-52.

8. Hernández Ramírez V. Relación entre el cáncer colorrectal y la enfermedad inflamatoria intestinal. Inflamatoria 2012; (7):21-9.

9. Meyer L, Simian D, Kronberg U, Estay C, et al. Desarrollo de neoplasia en pacientes con enfermedad inflamatoria intestinal. Rev Med Chile 2015;143(7):834-40. 